\title{
Para-phenylenediamine allergy: current perspectives on diagnosis and management
}

This article was published in the following Dove Press journal:

Journal of Asthma and Allergy

18 January 2017

Number of times this article has been viewed

\section{Krishna Sumanth \\ Mukkanna' \\ Natalie M Stone ${ }^{2}$ \\ John R Ingram ${ }^{3}$}

'Dermatology Department, Abertawe Bro Morgannwg University Health Board, Swansea, ${ }^{2}$ Dermatology

Department, Aneurin Bevan University

Health Board, Newport, ${ }^{3}$ Division

of Infection and Immunity, Cardiff

University, Cardiff, UK
Correspondence: Krishna Sumanth Mukkanna

Dermatology Department, Abertawe Bro Morgannwg University Health Board, Singleton Hospital, Sketty Lane, Sketty, Swansea SA2 8QA, UK

Tel +441792205666

Email ksumanth@doctors.org.uk
Abstract: Para-phenylenediamine (PPD) is the commonest and most well-known component of hair dyes. Oxidative hair dyes and dark henna temporary tattoos contain PPD. Individuals may be sensitized to PPD by temporary henna tattooing in addition to dyeing their hair. PPD allergy can cause severe reactions and may result in complications. In recent years, frequency of positive patch test reactions to PPD has been increasing. Cross-sensitization to other contact allergens may occur, in particular to other hair dye components. Hairdressers are at a high risk for PPD allergy and require counseling regarding techniques to minimize exposure and protective measures while handling hair dye. We focus this review on the current perspectives of diagnosis and management of PPD allergy.

Keywords: para-phenylenediamine, patch test, hair dye

\section{Introduction}

Para-phenylenediamine (PPD) was first described by Hofmann in $1863^{1}$ and formulated to use in hair dye at the end of the 19 th century. $^{2}$ It is a preferred constituent in many hair dyes in view of the longer lasting nature and black, natural appearing pigmentation imparted to the hair. ${ }^{2}$ PPD requires a secondary ingredient such as a developer or oxidizer to produce the black color. It is within the epidermis or dermis that PPD is oxidized to an allergenic hapten. ${ }^{3}$

\section{Epidemiology}

PPD has been identified as a common hair dye contact allergen for a number of years. Contact allergy to PPD can manifest as acute, subacute, or chronic dermatitis. Different studies involving the dermatitis patients have shown the median prevalence of positive PPD patch test to be $6.2 \%$ in North America, $4 \%$ in Europe, and $4.3 \%$ in Asia, although there may be broad variations within the country and between different countries. ${ }^{4-7}$ Patch test centers of Southern and Central Europe have a higher prevalence of PPD sensitization than Scandinavian countries, corresponding to the proportion of dark-haired individuals in the region. ${ }^{8}$ Lower prevalence in Scandinavia may be due to the higher proportion of blond-haired individuals. Darker shades of hair dye products contain higher concentrations of PPD and its related substances. ${ }^{9}$

There has been an increase in the frequency of positive patch test reactions to PPD over time, which could be due to larger number of people dyeing their hair, and at an increasingly earlier age. ${ }^{10}$ In India, PPD use was initially restricted to disguising 
the grey and white hairs among the older population; recent times have seen a greater use among the younger generation who would like to experiment with their looks. ${ }^{11}$ Hair dye dermatitis is thus showing a higher incidence among the whole population. ${ }^{11}$

The frequency of induction of contact allergy to PPD by black henna tattooing is estimated to be $2.5 \%$ per application. This trend has contributed to the increasing incidence of PPD allergy. After sensitization, patients may later manifest allergic contact dermatitis when using PPD-containing hair dyes. ${ }^{12}$ More recently, younger people consider temporary henna tattooing as a fashion. Black henna is a combination of PPD and natural henna, which intensifies the color as well as reduces application time. ${ }^{13}$ PPD is responsible for most of the allergic contact dermatitis and complications reported after temporary henna tattoos. ${ }^{14}$ For example, Haluk Akar et $\mathrm{al}^{15}$ reported an angioedema-like reaction following first time application of PPD-containing hair dye in a 15-year-old teenager who was not aware of previous sensitization to PPD from a black henna tattoo. Temporary henna tattoos are important source of exposure and sensitization to PPD, particularly in children, which may pose health and career consequences later in life. ${ }^{12}$

There are five different categories of hair dyes, each one having specific composition and mode of action: 1) gradual hair coloring (mainly metallic dyes, e.g., salts of silver, lead, and bismuth), 2) vegetable hair dyes (e.g., henna), 3) temporary hair dyes such as water-soluble hair dyes that resist only 1 shampooing, 4) semipermanent dyes (can resist 4 to 5 shampooings; may contain PPD-related chemicals), and 5) permanent or oxidation hair colors (usually PPD and/ or related chemicals), which is a very important category. ${ }^{16}$ Permanent hair dye compounds such as aromatic amines may cause contact allergy to self-hair dye users, hairdressers, and their clients. ${ }^{17,18}$ Hairdressers are usually exposed to both skin irritants and sensitizers; so, they are at a higher risk of occupational skin diseases. ${ }^{19}$

\section{Clinical presentation}

Topical PPD exposure may cause primary sensitization, resulting in subsequent allergic contact dermatitis. Skin and mucosal manifestations may differ, but they are usually characterized by pruritus, erythema, and vesicular or even bullous dermatitis in severely affected patients. ${ }^{20}$

Most reactions are delayed-type IV hypersensitivity reactions, and the symptoms and signs therefore have a latent period of several hours to days following the exposure to the hair dye. Pruritus, erythema, papules, and vesicles may involve the face, scalp margins (Figure 1 and 2) or ears. The patient provided written informed consent including

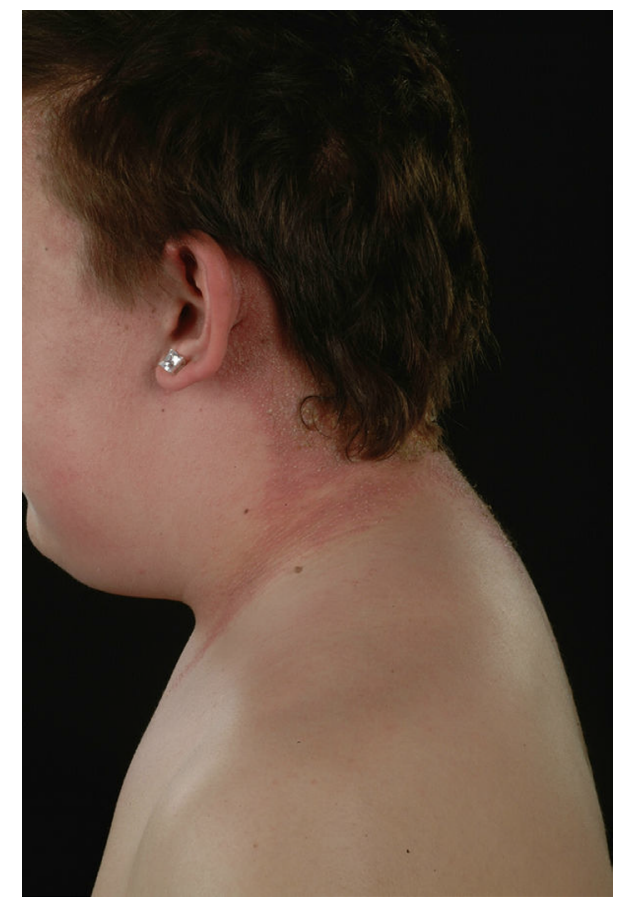

Figure I PPD allergy involving hairline. Abbreviation: PPD, para-phenylenediamine.

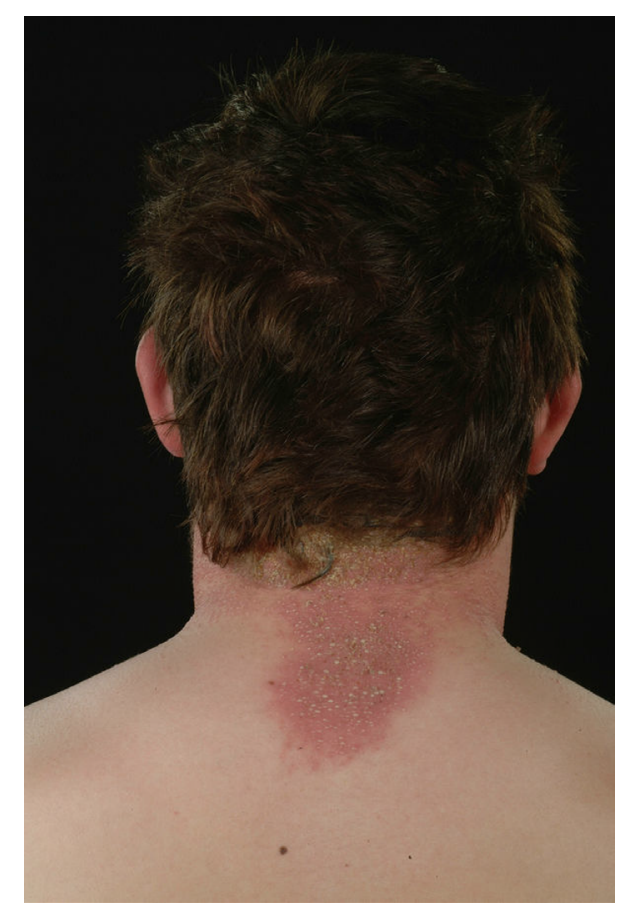

Figure 2 Posterior hairline involvement.

permission to use her pictures for this review. The scalp often fails to demonstrate visible inflammation, ${ }^{21}$ perhaps because it is shielded from dye by the hair itself. The severity of manifestations may vary from mild pruritus to prominent eyelid and facial edema, exudation, or blister formation. Systematization of the dermatitis can also occur involving other areas such as the neck, chest, arms, and, 
rarely, erythroderma. Men dyeing the hairs of moustache or beard may develop lesions on the central face. Several individuals may also notice photosensitivity. Few people may have predominantly airborne contact dermatitis-like manifestation as a presenting symptom with involvement of the eyelids, flexures such as antecubital fossa, and retroauricular folds. ${ }^{13}$

An angioedema-like appearance is also common and may lead to diagnostic confusion. The timing and history of hair dye exposure is, however, key to clarifying the diagnosis.

Earliest signs of allergic contact dermatitis can develop within 1-3 days of exposure to black henna tattoos in previously sensitized and within 4-14 days in non-sensitized patients. ${ }^{12}$ Post-inflammatory hypopigmentation is common, particularly in children, and can take more than 6 months to clear or may be permanent. ${ }^{12,14}$ Hyperpigmentation occurs mostly in adults and adolescents. ${ }^{12,14}$

PPD allergy can present with relatively rare skin manifestations, which are described in case reports, such as erythema multiforme, ${ }^{22}$ severe inflammatory, keloidal allergic reaction, ${ }^{23}$ contact urticaria, ${ }^{24}$ systemic manifestations such as acute renal failure, ${ }^{25}$ and even anaphylaxis. ${ }^{26}$

\section{Diagnosis}

Patch test

The gold standard test to confirm hair dye contact allergy is by epicutaneous patch test. PPD $1 \%$ in petrolatum (pet) is recommended to be tested as part of the standard European patch test series. Patch testing involves a small amount of commercially prepared allergen being placed in/on a chamber and applied to the back for 48 hours prior to removal. Patch tests are usually read on day 2 and day 4 to look for positive allergic reactions (Figure 3 ).

The oxidation of PPD on the surface and within the skin is responsible for the initiation of allergic reaction. Individuals with severe PPD reactions are more likely to provide a history of previous reaction to commercial hair dye. ${ }^{27}$ Those people sensitized by exposure to black henna tattoos and subsequently patch tested with standard $1 \%$ pet PPD often react strongly to PPD. ${ }^{28}$ Some centers now recommend testing patients with the lower $0.3 \%$ PPD concentration if they have a history of a black henna tattoo allergy in order to avoid a severe blistering patch test reaction. PPD 1\% patch testing can cause active sensitization. However, it is uncommon. ${ }^{27}$ Active sensitization to $1 \%$ PPD has been reported as a delayed reaction up to 15 days. ${ }^{29}$ Reactions later than 4 days could suggest the possibility that active sensitization events are occurring; however, this concept has been debated as several

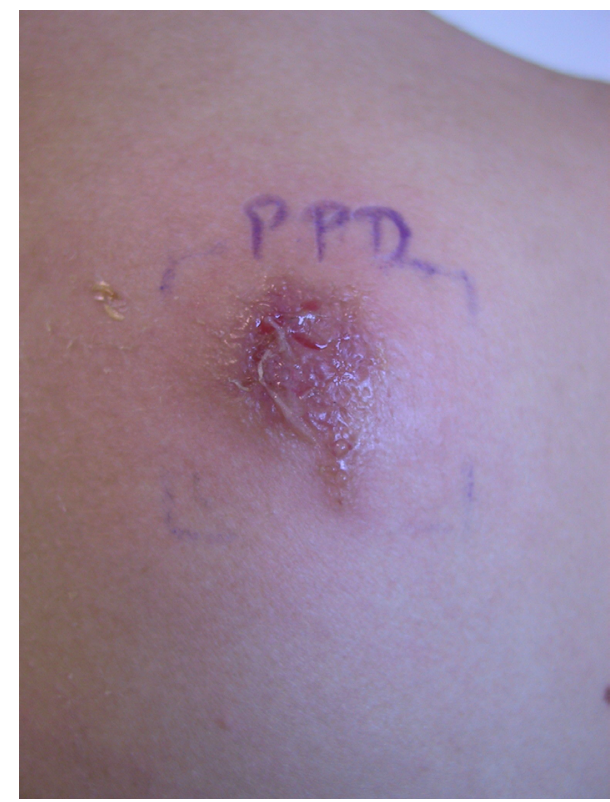

Figure 3 Blistered positive patch test reaction on day 4 to I\% PPD. Abbreviation: PPD, para-phenylenediamine.

true elicitation reactions can occur even after 7 days. ${ }^{30}$ Geier et $\mathrm{al}^{31}$ have recommended PPD $0.3 \%$ pet among the baseline series instead of PPD 1\% pet to decrease the risk of active PPD sensitization.

A human repeated insult patch test demonstrated that the sensitization rate of $1 \%$ PPD was reduced from $54 \%$ to $3 \%$ by decreasing the PPD exposure period from 48 hours to 5 minutes. However, another extended clinical trial demonstrated that higher concentration and infrequent but longer period of exposure to PPD was significantly less likely to cause sensitization on comparing with lower concentrations and more frequent but short period of PPD exposure. ${ }^{32}$

\section{Open test}

Open testing is recommended by hair dye manufacturers to test for allergic reactions 48 hours prior to hair dye applications. They suggest that a small amount of the dye is placed behind the ear and the skin be observed for redness.

Previous studies have reported the specificity and sensitivity of commercial open cutaneous allergy tests to anticipate the probability of hair dye allergic contact dermatitis. ${ }^{33,34}$ Thirty-four PPD-positive allergic patients and 49 non-allergic control individuals were investigated with test products of increasing concentrations of PPD $(0.1 \%, 0.5 \%, 1.0 \%$, or $1.5 \%$ ). It was noted that all PPD-allergic subjects developed positive reactions and no one from the control non-allergic subjects developed positive reactions. It was decided that the open test is suitable for pre-dyeing PPD allergy screening. It is possible, however, that this testing is adding further 
cutaneous exposure to PPD and may play a part in increasing the risk of PPD sensitization. The investigation will also miss patients who develop reactions beyond 48 hours, or who have weaker positive reactions that would only be detected by formal closed patch testing with pre-prepared allergens in diagnostic chambers.

\section{In vitro test}

Kneilling et $\mathrm{al}^{35}$ have described an in vitro lymphocyte activation test (LAT) for the diagnosis of PPD allergy. Titrated amounts of PPD without interleukin (IL)-2 or with IL-2 supplementation are cultured with freshly isolated peripheral blood mononuclear cells (PBMC). Proliferation of cells is determined by incorporation of $[3 \mathrm{H}]$-thymidine. ${ }^{35}$ LATs are used to identify PPD-specific PBMC cell proliferation, with a minimum of three and half fold raise in the uptake of [3H]thymidine needed for an individual to be labeled as allergic to PPD. PPD-LATs containing IL-2 supplementation have exhibited $100 \%$ sensitivity and remain unresponsive in PPD non-sensitized controls as well as in those sensitive to other similar p-amino compounds.

The in vitro LAT is an alternative to patch testing, particularly for severe PPD-allergic patients. ${ }^{35}$ However, the test is not widely available in clinical practice at the present time.

\section{Cross-reactivity}

PPD may show cross-sensitization with other compounds that also contain an amine group in their benzene ring at the para position (Table 1). PPD-allergic individuals exhibit a relatively high risk of concurrent sensitivity to other chemically related hair dyes as well as clothing dyes. ${ }^{27}$

There are case reports ${ }^{36}$ of hair dye allergy to PPD-free hair dye products because individuals can develop allergic reactions to other cross-reacting chemicals such as toluene2,5-diamine (PTD), which is commonly present in PPD-free products. Hence, PPD-allergic patients need clear information to make sure that they are not unintentionally exposed to risk due to potentially misleading labeling on hair dye packs. PPD-allergic patients who are confirmed on patch testing to

Table I Chemicals that cross-react with PPD

\begin{tabular}{ll}
\hline Chemical & Product \\
\hline Para-aminobenzoic acid & Sunscreens \\
Sulfonamides, sulfonylureas & Medications \\
Para-toluenediamine sulfate & Semipermanent hair dyes \\
Azo dyes & Clothes, ballpoint pen ink, shoes \\
Benzocaine, tetracaine, procaine & Local anesthetics \\
Para-aminosalicylic acid & Antibiotics \\
\hline
\end{tabular}

Abbreviation: PPD, para-phenylenediamine. be non-reactive to PTD may safely use a permanent hair dye, which contains only PTD as hair colorant. However, due to the presence of potentially cross-reactive colorants in most permanent hair dyes, the safest approach is to recommend only the non-permanent hair dyes.

A retrospective study ${ }^{37}$ of $2211 \%$ PPD patch test-positive patients showed an association between more vigorous reactions $(3+$ rather than $1+)$ and increased propensity to react to related compounds. An increasing likelihood of crossreactions to $\mathrm{N}$-isopropyl- $\mathrm{N}^{\prime}$-phenyl-p-phenylenediamine, Caine mix, and Disperse Yellow 3 was noted with increasing strength of patch test reactions to PPD; however, these crossreactions have an unclear clinical significance.

\section{Patch testing for strongly sensitized PPD patients}

The effective and safe way of confirming sensitization to PPD with reduced risk of severe reactions in strongly sensitized patients is either with PPD $0.3 \%$ pet for 2 days or $1 \%$ pet for 1 hour in those with a previous history of strong reaction to hair dyes. Patients who are not reactive to either test on day 2 can then be tested with 1\% PPD for the next 2 days. ${ }^{38}$

Patch test with 1\% PPD pet is often blistering type in patients with a past history of reaction to black henna tattoo. It has been recommended to test these patients at $0.01 \%$ PPD to reduce the chance of a severe reaction. ${ }^{28}$ An alternative approach is to minimize the contact time with the $1 \%$ concentration patch by advising patients to remove the PPD patch early if symptomatic, the patch being placed on an accessible part of the body such as the arm.

\section{Treatment}

The causative hair dye should be withdrawn. For acute, severely affected patients with PPD hair dye dermatitis, the scalp and hair should be washed thoroughly with a soap substitute to remove any excess dye. In theory, it may be useful to completely oxidize the PPD by applying hydrogen peroxide or potassium permanganate solutions; ${ }^{39}$ however, in practice, this is not tolerated by patients. Further treatment with a potent topical corticosteroid or short course of oral corticosteroids may be required as well as oral antibiotics if there is secondary skin infection. Oral antihistamines to counter the pruritus may also be considered.

\section{PPD-free alternative hair dyes}

Hydroxyethyl-p-phenylenediamine sulfate (HPPS) is a recognized sensitizer, which is tested as an alternative dyeing agent for PPD-sensitive individuals. ${ }^{40}$ In one report, $19.9 \%$ 
(40/216) individuals reacted to $1 \%$ PPD; however, only $0.9 \%$ $(2 / 216)$ were $1 \%$ HPPS positive and $2.3 \%$ of patients reacted to $2 \%$ HPPS.

Another alternative PPD derivative is 2-methoxymethylp-phenylenediamine. This is reported to be safe for use even in those with a $3+$ positive response to PPD. ${ }^{41}$

Selecting a synthetic formulation that is free of PPD may help allergic patients to avoid dermatitis. ${ }^{42}$ Herbal hair dye derivatives like henna generally have low allergic potential; however, some of them may contain diaminotoluenes and diaminobenzenes as additives, which can lead to contact dermatitis. ${ }^{43}$ Semipermanent hair dyes contain lower allergen concentrations; however, the dose-independent nature of allergic contact dermatitis means that reactions are still likely in sensitized individuals.

It should be noted that $57 \%$ of patients allergic to PPD were able to tolerate both newer permanent as well as demipermanent hair dyes (demipermanent dyes usually include ethanolamine and are moderately effective in coloring gray hair) if based on para-toluene diamine sulfate (PTDS) and PPD free. ${ }^{42}$ Most PTDS non-allergic patients also tested negative for other components of hair dye series. All 10 patients who were patch test PTDS negative and PPD positive later applied a PPD free PTDS dye and tolerated this well. Many PPD-allergic patients will benefit from these newer PTDS-based hair dye products. However, $43 \%$ of those tested had positive reactions and so patients should be made aware that comprehensive prior patch testing is required and the alternative hair colorant may not be suitable for them, depending on the results.

Patients need clear advice that it is easiest to simply avoid all permanent and semipermanent hair dyes in future once diagnosed with PPD allergy. The wash in-wash out dyes are usually safe.

\section{Future/Prevention}

Keeping the PPD concentration to the basic minimum level in hair dyes at the manufacturing level is an important consideration for hair dye producers. Restriction to PPD-free hair dyes (also avoiding potentially cross-reacting dyes) and vegetable-based hair dye is perhaps the best practical solution for those exhibiting PPD sensitization. Hair stylists and patients should be advised to use gloves while applying hair dyes to prevent contact with hands.

In an Australian study ${ }^{44}$ patients diagnosed with PPD allergy experienced a significant disturbance in their lifestyle. The study highlighted that standard medical advice of "stop the use of hair dye" is one dimensional and patients may not follow the advice. In all, $78 \%$ of patch test-diagnosed
PPD-allergic patients tried alternative hair dyes and greater than $50 \%$ continued hair dyeing. The majority of PPD-allergic patients can find the commercial hair dye market confusing as they commonly obtain information either from friends, family members, hair stylists, or chat forums on Internet. Confusion is not surprising due to 'natural', 'organic' or 'pure' marketing claims for dyes that often contain aromatic amines, which may cross-react with PPD. It is very important to advise all the patients to read the ingredient list instead of relying on the product name in order to identify other potential cross-reactants within the ingredient list. Patients need careful written advice about checking product ingredients, always using open patch tests correctly, and avoiding products if there is any doubt.

It is helpful to involve an experienced and well-trained hairdresser who uses proper techniques when applying hair dye. During hair dye self-application, it can be difficult to minimize spread onto the scalp. Before hair dye application, to minimize lateral spread of the hair dye and to protect the scalp margin, hairdressers can apply a barrier of pet. ${ }^{45}$ Recommended approaches for hairdressers to prevent hand dermatitis are proper education, appropriate use of gloves, and emollient use to prevent irritant hand eczema.

\section{Conclusion}

PPD is the commonest cause of hair dye contact dermatitis. Patients can present with a variety of clinical manifestations, including severe reactions. Patch testing remains the gold standard method for confirming PPD allergy. Avoiding future contact with permanent and semipermanent hair dyes is the standard preventative measure. A few PPD-allergic patients can successfully continue hair dyeing if comprehensive patch testing is undertaken and detailed written advice is provided for the individual regarding safe and unsafe hair dye components, including the potential for cross-reactivity. Further work is needed to raise public awareness that black henna tattoos are an important source of PPD sensitization.

\section{Acknowledgment}

The authors are thankful to Dr S Blackford for providing clinical photographs.

\section{Disclosure}

The authors report no conflicts of interest in this work.

\section{References}

1. Hofmann AW. Organische Basen. Jahresberichte ueber die Fortschritte der Chemie. [Annual report of progress in chemistry]. 1863;3:422. German. 
2. Colour and controversy. The Royal Society of Chemistry website. Available from: http://www.rsc.org/chemistryworld/2012/09/hair-dyeppd-allergen-regulation. Accessed March 13, 2016.

3. Mohamed M, Nixon R. Severe allergic contact dermatitis induced by paraphenylenediamine in paint-on temporary 'tattoos'. Australas $J$ Dermatol. 2000;41(3):168-171.

4. Thyssen JP, White JM; European Society of Contact Dermatitis. Epidemiological data on consumer allergy to p-phenylenediamine. Contact Dermatitis. 2008;59(6):327-343.

5. Chan YC, Ng SK, Goh CL. Positive patch-test reactions to paraphenylenediamine, their clinical relevance and the concept of clinical tolerance. Contact Dermatitis. 2001;45(4):217-220.

6. Sharma VK, Chakrabarti A. Common contact sensitizers in Chandigarh, India. A study of 200 patients with the European standard series. Contact Dermatitis. 1998;38(3):127-131.

7. Sharma VK, Sethuraman G, Garg T, Verma KK, Ramam M. Patch testing with the Indian standard series in New Delhi. Contact Dermatitis. 2004;51(5-6):319-321.

8. Thyssen JP, Andersen KE, Bruze M, et al. p-Phenylenediamine sensitization is more prevalent in central and southern European patch test centres than in Scandinavian: results from a multicentre study. Contact Dermatitis. 2009;60(6):314-319.

9. Vogel TA, Coenraads PJ, Bijkersma LM, Vermeulen KM, Schuttelaar ML; EDEN Fragrance Study Group. p-Phenylenediamine exposure in real life - a case-control study on sensitization rate, mode and elicitation reactions in the northern Netherlands. Contact Dermatitis. 2015;72(6): 355-361.

10. Patel S, Basketter DA, Jefferies D, et al. Patch test frequency to p-phenylenediamine: follow up over the last 6 years. Contact Dermatitis. 2007;56(1):35-37.

11. Sudhir Nayak UK. Hair dye dermatitis and para-phenylenediamine contact sensitivity. Indian Dermatol Online J. 2015;6(4):246-247.

12. de Groot AC. Side-effects of henna and semi-permanent 'black henna' tattoos: a full review. Contact Dermatitis. 2013;69(1):1-25.

13. Handa S, Mahajan R, De D. Contact dermatitis to hair dye: an update. Indian J Dermatol Venereol Leprol. 2012;78(5):583-590.

14. Kazandjieva J, Grozdev I, Tsankov N. Temporary henna tattoos. Clin Dermatol. 2007;25:383-387.

15. Haluk Akar H, Adatepe S, Tahan F, Solmaz I. Hair dyes and temporary tattoos are a real hazard for adolescents? Eur Ann Allergy Clin Immunol. 2014;46(1):35-37.

16. Boldoc C, Shapiro J. Hair care products: waving, straightening, conditioning and coloring. Clin Dermatol. 2001;19(4):431-436.

17. Leino T, Estlander T, Kanerva L. Occupational allergic dermatoses in hairdressers. Contact Dermatitis. 1998;38(3):166-167.

18. Søsted H, Agner T, Andersen KE, Menné T. 55 cases of allergic reactions to hair dye: a descriptive, consumer complaint-based study. Contact Dermatitis. 2002;47(5):299-303.

19. Uter W, Lessmann H, Geier J, Schnuch A. Contact allergy to ingredients of hair cosmetics in female hairdressers and clients - an 8-year analysis of IVDK data. Contact Dermatitis. 2003;49(5):236-240.

20. Goldenberg A, Jacob SE. Paraphenylenediamine in black henna temporary tattoos: 12-year Food and Drug Administration data on incidence, symptoms, and outcomes. J Am Acad Dermatol. 2015;72(4):724-726.

21. Zhai H, Zheng Y, Fautz R, Fuchs A, Maibach HI. Reactions of nonimmunologic contact urticaria on scalp, face, and back. Skin Res Technol. 2012;18(4):436-441.

22. Barrientos N, Abajo P, de Vega MM, Dominguez J. Erythema multiforme-like eruption following allergic contact dermatitis in response to para-phenylenediamine in a temporary henna tattoo. Int $J$ Dermatol. 2014;53(7):e348-e350.

23. Gunasti S, Aksungur VL. Severe inflammatory and keloidal, allergic reaction due to para-phenylenediamine in temporary tattoos. Indian $J$ Dermatol Venereol Leprol. 2010;76(2):165-167.
24. Pasche-Koo F, French L, Piletta-Zanin PA, Hauser C. Contact urticaria and shock to hair dye. Allergy. 1998;53(9):904-905.

25. Abdelraheem MB, El-Tigani MA, Hassan EG, Ali MA, Mohamed IA, Nazik AE. Acute renal failure owing to paraphenylene diamine hair dye poisoning in Sudanese children. Ann Trop Paediatr. 2009;29(3): 191-196.

26. Belton AL, Chira T. Fatal anaphylactic reaction to hair dye. Am J Forensic Med Pathol. 1997;18(3):290-292.

27. McFadden JP, Yeo L, White JL. Clinical and experimental aspects of allergic contact dermatitis to para-phenylenediamine. Clin Dermatol. 2011;29(3):316-324.

28. Ho SG, White IR, Rycroft RJ, McFadden JP. A new approach to patch testing patients with para-phenylenediamine allergy secondary to temporary black henna tattoos. Contact Dermatitis. 2004;51(4):213-214.

29. Le Coz CJ, El Bakali A, Untereiner F, Grosshans E. Active sensitization to budesonide and para-phenylenediamine from patch testing. Contact Dermatitis. 1998;39(3):153-155.

30. Gawkrodger DJ, Paul L. Late patch test reactions: delayed immune response appears to be more common than active sensitization. Contact Dermatitis. 2008;59(3):185-187.

31. Geier J, Ballmer-Weber BK, Dickel H, et al. Monitoring contact sensitization to p-phenylenediamine (PPD) by patch testing with PPD $0.3 \%$ in petrolatum. Contact Dermatitis. 2013;69(1):26-31.

32. Basketter DA, Jefferies D, Safford BJ, et al. The impact of exposure variables on the induction of skin sensitization. Contact Dermatitis. 2006; 55(3): 178-185.

33. Krasteva M, Cottin M, Cristaudo A, et al. Sensitivity and specificity of the consumer open skin allergy test as a method of prediction of contact dermatitis to hair dyes. Eur J Dermatol. 2005;15(1):18-25.

34. Krasteva M, Cristaudo A, Hall B, et al. Contact sensitivity to hair dyes can be detected by the consumer open test. Eur J Dermatol. 2002;12(4):322-326.

35. Kneilling M, Caroli U, Grimmel C, et al. Para-phenylenediaminespecific lymphocyte activation test: a sensitive in vitro assay to detect para-phenylenediamine sensitization in patients with severe allergic reactions. Exp Dermatol. 2010;19(5):435-441.

36. Ingram JR, Hughes TM, Stone NM. Potential danger of hair dyes marketed as free from para-phenylenediamine. Int J Dermatol. 2014; 53(4):e257-e258.

37. Thomas BR, White IR, McFadden JP, Banerjee P. Positive relationshipintensity of response to $\mathrm{p}$-phenylenediamine on patch testing and crossreactions with related allergens. Contact Dermatitis. 2014;71(2):98-101.

38. Nalluri R, Williams J. Para-Phenylenediamine testing in strongly sensitized patients: an effective screen. Br J Dermatol. Presented at: 94th Annual Meeting of the British Association of Dermatologists; $2014 \mathrm{Jul}$ 1-3; Glasgow; 2014;171(Suppl 1):133.

39. Allergy to paraphenylenediamine. DermNet New Zealand Trust website. Available from: http://www.dermnetnz.org/topics/allergy-toparaphenylenediamine/. Accessed September 21, 2016.

40. Frosch PJ, Kügler K, Geier J. Patch testing with hydroxyethyl-pphenylenediamine sulfate - cross-reactivity with p-phenylenediamine. Contact Dermatitis. 2011;65(2):96-100.

41. Edwards A, Coman G, Blickenstaff N, Maibach H. Dermatotoxicologic clinical solutions: hair dying in hair dye allergic patients? Cutan Ocul Toxicol. 2015;34(1):72-74.

42. Scheman A, Cha C, Bhinder M. Alternative hair-dye products for persons allergic to para-phenylenediamine. Dermatitis. 2011;22(4):189-192.

43. Polat M, Dikilitaş M, Oztaş P, Alli N. Allergic contact dermatitis to pure henna. Dermatol Online J. 2009;15(1):15.

44. Jenkins D, Chow ET. Allergic contact dermatitis to para-phenylenediamine. Australas J Dermatol. 2015;56(1):40-43.

45. Ashworth J, Watson WS, Finlay AY. The lateral spread of clobetasol 17-propionate in the stratum corneum in vivo. Br J Dermatol. 1988; 119(3):351-358. 
Journal of Asthma and Allergy

The Journal of Asthma and Allergy is an international, peer-reviewed open access journal publishing original research, reports, editorials and commentaries on the following topics: Asthma; Pulmonary physiology; Asthma related clinical health; Clinical immunology and the immunological basis of disease; Pharmacological interventions and new therapies. This journal is included in PubMed. The manuscript management system is completely online and includes a very quick and fair peer-review system, which is all easy to use. Visit http://www. dovepress.com/testimonials.php to read real quotes from published authors.

Submit your manuscript here: https://www.dovepress.com/journal-of-asthma-and-allergy-journal 